\title{
Vowels Variation of Interrogative 'Which' in Northern and Central Tai
}

\author{
Yingzhu Chen ${ }^{1,}$ Yan Zhang ${ }^{2 *}$
}

\author{
${ }^{1}$ Guangxi University of Chinese Medicine \\ ${ }^{2}$ Guangxi University of Chinese Medicine \\ ${ }^{*}$ Yan Zhang. Email: zhangyancncn@163.com
}

\begin{abstract}
This paper investigates the vowels of interrogative "which" in more than 20 dialects in contemporary Tai, and analyses the geographical distribution patterns. The related phonological variation chains of Kam-Tai languages and other languages families provide some clues that the vowels of "which" in Northern and Central Tai dialects are partly consistent. Internal factors play the major role on the vowel variation of 'which', while the influence of language contact cannot be excluded. To some extent, the vowel variation patterns of this interrogative confirm the diachronic reconstruction, and reflect the relationship between space and time (synchronic and diachronic).
\end{abstract}

Keywords: Vowels variation, Tai interrogative “which”, Geographical distribution.

\section{INTRODUCTION}

Phonological variation is related to geographical distribution. The distinction of language in different areas may be the remains in different historical stages [1]. Pan [2] argues that real time sound shifts in the history of a language from the geographical distribution reflect apparent time sound shifts. He [3] further states that the synchronic distinction can probably reflect diachronic change of languages. That is to say, the geographical distribution might be the result of language change.

Preliminary studies focus on phonological variation in a particular group of vowels in Zhuang and Kam-Tai languages [4-7]. However, no previous studies have been attempted on the highly frequently used function words, interrogatives. As one of the most active interrogatives, the patterns of phonological variations of 'which' in Tai had yet to be found. Thus, the purpose of this paper is to fill this gap by examining the directions of phonological variation of 'which' in detail and determining the linguistic factors that are correlated with the sound variations.

This paper reports the investigation results of the vowel change of the interrogative 'which' in more than 20 dialects of Tai to answer the following two questions:
(1) What are the synchronic geographical distribution features of the vowels of "which" in Northern and Central Tai dialects? (2) What is the direction of the variation pattern of the vowels of "which" in the Northern and Central Tai dialects?

\section{DATA}

Mostly the Zhuang people in southwest China speak Tai language. The Tai language family is taken as a major sub-branch of Kam-Tai within the greater grouping Tai-Kadai. According to Li [5], Tai languages are divided into three subgroups: Northern, Central and Southwest groups.

The data used in the present paper cover a broad geographical area. Figure 1 is a map of all dialects included in this study, mainly located in Guangxi Zhuang Autonomous Region.

The varieties included in this paper belong to all two dialectal groups: Central Tai and Northern Tai. Debao, Jingxi, Daxin, Longchow, Ningming, Pingxiang, Shangsi, Shuangding dialects are Central branch. Rong'an, Liucheng, Liujiang, Xincheng, Fengshan, Donglan, Bma, Du'an Mashan, Tianyang, Pinguo and Wuming dialects are Northern branch. 


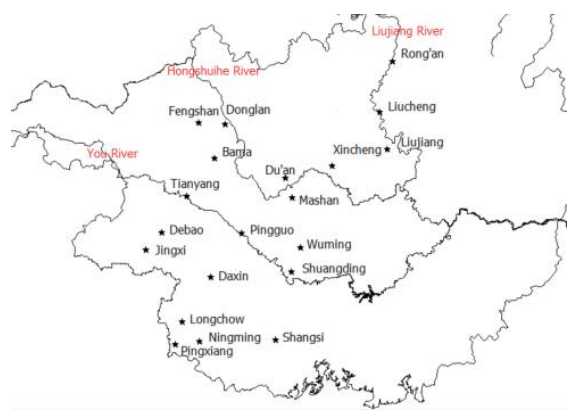

Figure 1 Northern and Central Tai dialects included in this study.

\section{GEOGRAPHICAL DISTRIBUTION PATTERN}

Geographical factors, such as mountains and rivers, are known to have certain effects on language variation. [1]. In terms of phonological variation, there are four major patterns [8]: consistent, discrete, recursive, and radiation. The consistent pattern refers to some variation features that exist in several adjacent dialects; the discrete pattern is the variation distributed sporadically in the dialects; the recursive pattern refers to the increase in the variation feature from north to south; and the radiation pattern is the variation radiating from the centre, like a wave shape.

There are six vowels of 'which' being observed in this paper: /ə/, /əu/, /aw/, /ai/, /əi/, /ou/.

The vowels of 'which' in Northern and Central Tai dialects show the following three geographical distribution features.

\subsection{Consistent Pattern}

In the first place, the vowels distribution of "which" is divided by the middle of Xincheng, with /a/ distributing from the northern part of Xincheng to the northeast part, which shows the characteristics of a consistent pattern.

\subsection{Radiation Pattern}

However, /a/ does not exist from the southern part of Xincheng to the southwestern part of Guangxi. In the second place, between the area of the southwestern part of Xincheng and the You River, there is a concentric circle with three layers, /ow/, /aw/ and /ai/. The third layer of /ai/ is not a perfect circle, but a long belt along the You River.

What's more, in the southwestern part of the You River, a similar concentric circle with three layers exists, /ow/, /aw/ and /oi/. We can see that the difference between the two circles in Northern Tai and Central Tai is the third layer. Note that the vowel of 'which' in Ningming has two sounds, /ow/ and /aw/. Therefore, the first layer and the second layer in this county are overlapped. The three geographical distribution features of vowels of 'which' in the Northern and Central Tai dialects are shown in the Figure 2.

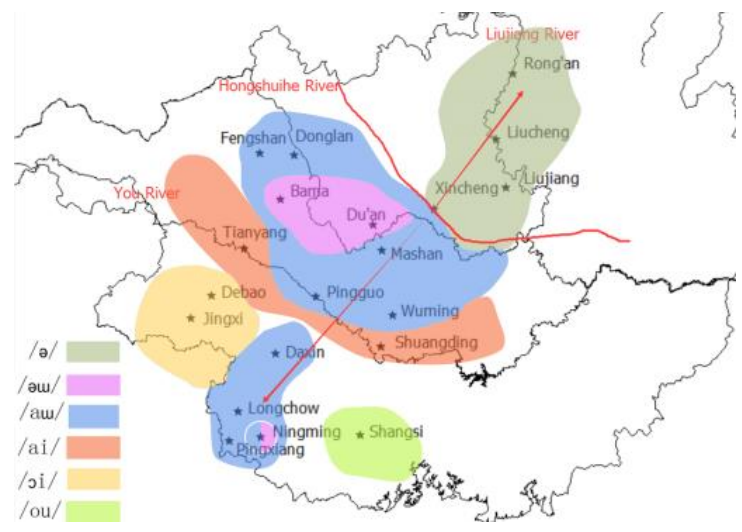

Figure 2 The geographical distribution features of vowels of 'which' in Northern and Central Tai.

\section{DISCUSSION}

From the phonological point of view, the vowels of 'which' in Northern Tai and Central Tai show distinctive features in the geographical distribution. Since the variation may not happen solely in one particular sound, and we may get some clues from the related chain shifts in the relative languages and nonrelative languages. This chapter will also try to figure out the main factors of vowels variations and to find out the variation patterns of 'which'. Presumably, the chain shifts may lead us to consider the directions of phonological variations and provide novel insights into the contemporary phonology. Then we can propose and analyze variation patterns in 'which' in Northern and Central Tai.

\subsection{Related Variation Chain Shifts}

The following part will focus on the chain shifts of vowels related to 'which' in Northern and Central Tai. We try to figure out whether or not the common chain shifts in various languages can provide a direction for the variation of 'which' in Northern and Central Tai. Based on the investigation on Northern and Central Tai dialects, Tai languages and Kai-Tai languages, some scholars proposed chain shifts of vowels.

According to Zhang [9], three vowels /w/, /ow/, /aw/ in Zhuang dialects interact with each other. The shifting trend of the three vowels is simplified to a typical chain shift, /aw/ $\rightarrow$ /ai/ or /oi/. It is found that the sound /ə/ in a large number of dialects in Kam-Tai languages comes from /əu/, that is, /əu/ $\rightarrow / \partial /$ [10]. Jin [11] argues that the sound /u/ in Kam-Tai languages will diphthongize into /aw/ and then /o/. Furthermore, the vowels in KamTai languages share a chain shift (see Figure 3 ) [12]. 
Because the sounds /ə/, /ow/, /aw/, /ai/, /oi/ can be found in the related vowel chain shifts, we also speculate that these vowels in 'which' in Northern and Central Tai transform in the similar ways. Therefore, [u] $\rightarrow / \mathrm{o} /,[\mathrm{u}] \rightarrow / \mathrm{ow} / \rightarrow / \mathrm{aw} / \rightarrow$ /ai/, and $[\mathrm{w}] \rightarrow /$ ow/ $\rightarrow /$ aw/ $\rightarrow / \mathrm{j}$ / are possible variation patterns of 'which'.

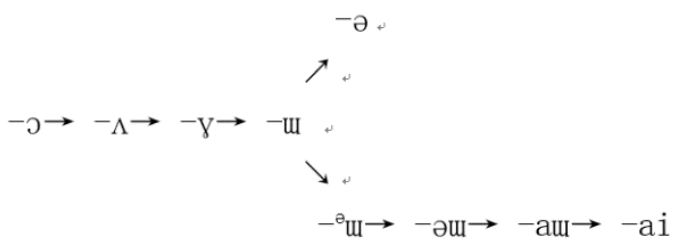

Figure 3 The vowel chain shift of Kam-Tai languages [12]

\subsection{The Influence of Contacts}

Zhuang people contact with the people who speak various dialects for a long time. First, we need to consider the influence of Chinese history in earlier times. In addition, since Qin Shihuang's southern expansion, non-Han peoples have predominantly inhabited Guangxi $[6,13]$.

The Han people moved from the Central Plains of China, integrating with the people in Guangxi. Intermarriages took place between local women and Han soldiers who settled down in the area, forming an independent dialect called Pinghua. In the Ming and Qing dynasties, especially after opening the country to foreign trade in the last two or three hundred years, Cantonese and Southwestern Mandarin started to enter Guangxi.

All these several languages and dialects have greatly influenced Northern and Central Tai. When we discuss the sound variation of "which" in Northern and Central Tai, their influence cannot be excluded.

\subsection{Proposed Variation Pattern}

Above all, related chain shifts in Northern and Central Tai, Kam-Tai and other world languages indicate that the same sound change occurs in other languages, which provides the direction as a reference for the variation of 'which' in Northern and Central Tai. Furthermore, the principles of natural processes offer the underlying factors for phonological variation.

Based on a close examination of the vowels of 'which' in Northern and Central Tai, the variation patterns are proposed, as shown in Figure 4, respectively.

Note that /ou/ in the Shangsi dialect does not exist in the proposed vowel variation pattern, for /ou/ in Tai languages may develop a path parallel with /ow/, and they may not interact with each other. We might assume that /ou/ may be influenced by language contact. The sounds in square brackets are not to be found in published materials, but may exist in some dialects. This needs to be further confirmed.

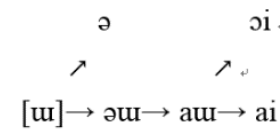

Figure 4 The vowels variation pattern of 'which' in Northern and Central Tai

\section{CONCLUSION}

In sum, this paper investigates the sound of 'which' in more than 20 Northern and Central Tai dialects. The vowels of 'which' have been centralized from northern part of Xincheng to northeast, and diphthongized from the southern part of Xincheng to the southwest. There are two concentric circles with three layers in the northeast part and southwest part of the You River, respectively. The vowels distribution in Northern Tai and in Central Tai is different, which show typological significance.

Based on related chain shifts in related languages and the external influence of contact, we proposed a vowel variation pattern of 'which' in Northern and Central Tai. The results may support the argument that synchronic distinction reflects diachronic change of languages.

\section{REFERENCES}

[1] Zhiyun Cao, Geographical linguistics and its development in China. The Journal of Chinese Dialects, vol.1, 2006, pp. 171-184.

[2] Wuyun Pan, Reconstruct real time sound shifts from the geographical apparent time sound changes. Minzu Yuwen, vol.32, 2010a, pp. 3-12. DOI:CNKI:SUN:MZYW.0.2010-01-003.

[3] Wuyun Pan, The historical development of Chinese dialects from perspective of geographical information. The Journal of Chinese Linguistics, vol. 45, 2017, pp. 458-465.

[4] Jahua Yuan, The correspondence of $/ \mathrm{r} /$ patterns on Zhuang. Anthology on Linguistics, vol. 5, 1963, pp. 187-218.

[5] Fangkuei Li, A Handbook of Comparative Tai. Oceanic Linguistics Special publications, vol. 15, 1977, pp. 1-389.

[6] Junru Zhang. The influence of Pinghua on KamTai languages in Guangxi). Minzu Yuwen, vol. 10, 1988, pp. 51-56. DOI:CNKI:SUN:MZYW.0.198803-009. 
[7] Yuzhu Zhu, A study on /r/ pattern in Northern Zhuang. MA thesis. Beijing: Minzu University of China,2013.

https://kns.cnki.net/KCMS/detail/detail.aspx?dbna $\mathrm{me}=\mathrm{CMFD} 201401 \&$ filename $=1013316042 . \mathrm{nh}$

[8] Wei Zheng, The Law of Sound Change and the Geographical Distribution Types of Chinese Dialects). Anthology on Language Studies, vol. 27, 2013, pp. 266-280+336-337. DOI:CNKI:SUN:YJJK.0.2013-02-023.

[9] Junru Zhang, The change of /u/, /ow/, /aw/ in Zhuang). Minzu Yuwen, vol. 7, 1986, pp. 20-24. DOI:CNKI:SUN:MZYW.0.1986-06-005.

[10] Min, Liang, Junru, Zhang, An introduction of KamTai language), 58. China Social Sciences Publishing House, Beijing, 1996.

[11] Lixin Jin, Long and Short Vowels in the Kam-Tai Languages). Studies in Language and Linguistics, vol. 31, 2011, pp. 42-56. DOI:CNKI:SUN:YYYJ.0.2011-04-010.

[12] Wei Zheng, The Chronological and Phonological Strata of $\mathrm{Yu}$ in Min Dialect and Kam-Tai Languages. Linguistic Science, vol. 7, 2008, pp. 320-328. DOI:CNKI:SUN:YYKE.0.2008-03-013.

[13] Yongxian Luo, Zhuang. The Tai-Kadai Languages, in: Anthony V. N. Diller (Eds.), Routledge, London, 2008, pp. 317-377. 